\title{
TOTAL STABILITY OF SETS FOR NONAUTONOMOUS DIFFERENTIAL SYSTEMS
}

\author{
ZHIVKO S. ATHANASSOV
}

\begin{abstract}
The principal purpose of this paper is to present sufficient conditions for total stability, or stability under constantly acting perturbations, of sets of a sufficiently general kind for nonautonomous ordinary differential equations. To do this, two Liapunov-like functions with specific properties are used. The obtained results include and considerably improve the classical results on total stability of isolated equilibrium points. Applications are presented to study the stability of nonautonomous Lurie-type nonlinear equations.
\end{abstract}

"Who said: two are enough?" Günter Grass, The Flounder

1. Introduction. The concept of stability is one of those which reaches beyond the general domain in mathematics. In his well-known dissertation Problème général de la stabilité du mouvement, Liapunov [1] has given several criteria for stability and asymptotic stability of solutions of systems of differential equations with the help of certain auxiliary scalar functions which are now commonly called Liapunov functions. The problem of stability and relationships between stabilities and Liapunov functions has been discussed by many authors since Liapunov.

The classical Liapunov theorem on asymptotic stability of the zero solution for a differential equation $\dot{x}=f(t, x)$ uses a positive definite function $V(t, x)$ whose time derivative $\dot{V}(t, x)$ along solutions of the differential equation has to be negative definite. However, this stronger property is not always possessed by the natural candidate for the Liapunov function, namely, the energy. The damped harmonic oscillator is the standard example.

An effective tool to overcome the difficulty was developed by LaSalle, and is usually referred to as the invariance principle. By combining information on level sets of the Liapunov function with the topological dynamics of the solutions flow, it is often possible to detect the $\omega$-limit set and thus establish stability properties. The technique is well documented in the literature and the reader may consult LaSalle [2] as a reference.

A different approach was proposed by Matrosov [3] who proved a group of theorems which are, so to say, of a more voluntary character. The purpose of these theorems is, given an auxiliary function $V(t, x)$, to find a second function $W(t, x)$ with suitable properties in the vicinity of the set, where $\dot{V}(t, x)=0$, in order to force the solutions to tend to zero as $t \rightarrow \infty$. The technique of proof is using $V$ and $W$ to hunt down the solution until it enters a suitable neighborhood of zero.

Received by the editors March 20, 1985.

1980 Mathematics Subject Classification. Primary 34D10, 34D20; Secondary 93C20, 93D20.

Key words and phrases. Total stability of sets, nonautonomous differential systems, Liapunovlike functions, Lurie-type equations, uniform asymptotic stability, absolute stability. 
No use is made of the properties of the limit sets. For some generalizations of the methods of LaSalle and Matrosov and comments on how they complement each other see Rouche et al. [4]. The methods of Liapunov, LaSalle and Matrosov are direct methods; i.e., one should be able to discover the stability by looking at the equation and without, for instance, computing the solutions.

The concepts of stability and asymptotic stability introduced by Liapunov could be called stabilities under sudden perturbations. The perturbation suddenly moves the system from its equilibrium state but then immediately disappears. Stability says that the effect of this will not be great if the perturbation is not too great. Asymptotic stability states, in addition, that if the sudden perturbation is not great, the effect of the perturbation will tend to disappear. In practice, however, the perturbations are not simply impulses and this led Duboshin [5] to consider what he called stability under constantly acting perturbations, today known as stability. This says that if the perturbation is not too large and if the system is not too far from the origin initially it will remain near the origin. Total stability can be described roughly as the property that a bounded perturbation has a bounded effect on the solution. There is a large amount of literature on applications of Liapunov functions to the study of perturbations; see, e.g., [6-9] and the references therein. Indeed, the study of perturbations is one of the motivations for studying Liapunov theory.

Consider the system defined by the differential equations

$$
\dot{x}=(x-y)\left(1-a^{2} x^{2}-b^{2} y^{2}\right), \quad \dot{y}=(x+y)\left(1-a^{2} x^{2}-b^{2} y^{2}\right)
$$

in the Euclidean plane (see $[10$, p. 18]). The phase curves approach the ellipse $a^{2} x^{2}+b^{2} y^{2}=1$ from the outside and from the inside depending on the sign of the time derivative of the Liapunov function $V(x, y)=x^{2}+y^{2}$. The ellipse itself is not a solution of the system.

As another example, consider the nonautonomous two-dimensional system

$$
\dot{x}=-y, \quad \dot{y}=x-(2+\exp t) y .
$$

The solution $x=1+\exp (-t), y=\exp (-t)$ approaches the point $(1,0)$ as $t \rightarrow \infty$, but $x=1, y=0$ is not a solution of this system.

The above examples indicate that a family of solutions of a differential equation may approach asymptotically a curve which need not be a solution of the differential equation. This motivates the study of the stability of sets as opposed to the stability of equilibrium points.

The stability of sets which includes, as special cases, the stability in the sense of Liapunov and the orbital stability is one of the most important problems in stability theory. The literature abounds with treatments of this problem; see Zubov [11] and Bhatia and Szegö [12] for details and further references, and Sestakov $[13,14]$ for references on the latest investigations in this field. By long-established tradition, asymptotically stable sets are compact or have compact closure of complement, i.e., are either very small or very large and the majority of the papers and books deal with such sets. There are numerous counterexamples to this: e.g., the $x$-axis for $\dot{x}=0, \dot{y}=-y$ or for $\dot{x}=x, \dot{y}=-y$. The classical theorems on the stability theory of equilibrium states may be extended without difficulty to the study of the stability of compact sets. However, when one begins to consider the stability of 
noncompact sets this extension is not trivial; see, for example, LaSalle and Szegö [15], LaSalle [16], Yoshizawa [17] and Hájek [18, 19].

In this paper we consider the problem of total stability of sets of a sufficiently general kind belonging to some domain (open, connected set) in which a nonautonomous system of nonlinear differential equations is given. We extend and push Matrosov's ideas further, to include total stability of sets. A major role in our techniques is played by two Liapunov-like functions whose properties are connected in a specific way with the notions of definiteness and existence of an infinitesimal upper bound of a function with respect to a given set. Our main results are further development and generalization of the classical theorems of Malkin [20] and Gorshin [21] of total stability for an isolated equilibrium state.

The organization of the present paper is as follows. In $\S 2$, we introduce some notation and the relevant definitions which will be used throughout the paper. The main results are presented in $\S 3$. We give three theorems pertaining to the total stability of sets. Sufficient conditions of total stability of a set with respect to a nonautonomous differential system are given in the first theorem. This is followed by several comments. The second theorem shows that the obtained sufficient conditions ensure some kind of asymptotic property. The third theorem is a modification of the first one. Applications to the stability of nonautonomous Lurie-type nonlinear equations are presented in the final section.

2. Notation and definitions. In order to state our results we need some notation and definitions. Consider the nonperturbed ordinary differential equation

$$
\dot{x}=f(t, x) \quad(\cdot=d / d t)
$$

where $x$ and $f$ belong to Euclidean $n$-space $R^{n}$ with Euclidean norm $|\cdot|, t$ is a real scalar and $f$ is defined and sufficiently smooth on the semicylinder

$$
D_{r}=\{(t, x): t \geq 0,|x|<r, r>0\}
$$

for the existence of all solutions. We denote by $x\left(t, t_{0}, x_{0}\right)$ a solution of $(\mathrm{N})$ through $\left(t_{0}, x_{0}\right) \in D_{r}$, thus displaying its dependence of initial conditions. By definition, $x\left(t_{0}, t_{0}, x_{0}\right)=x_{0}$. Another differential equation will have to be considered along with $(\mathrm{N})$, namely, the perturbed equation

$$
\dot{y}=f(t, y)+g(t, y)
$$

where $g: D_{r} \rightarrow R^{n}$ satisfies the same regularity conditions as $f$, thus ensuring existence for all solutions of $(\mathrm{P})$. This function $g$ will play the role of a perturbation term added to the second member of $(\mathrm{N})$. Not much will be supposed about it except these regularity conditions and, of course, some kind of bound. For $\left(t_{0}, x_{0}\right) \in D_{r}$, let $y\left(t, t_{0}, x_{0}\right)$ be that solution of $(\mathrm{P})$ for which $y\left(t_{0}, t_{0}, y_{0}\right)=y_{0}$.

If $z_{1}$ and $z_{2}$ are two points of $R^{n}$ and $G$ is a subset of $R^{n}$, we adopt the usual distances $d\left(z_{1}, z_{2}\right)=\left|z_{1}-z_{2}\right|$ and $d\left(z_{1}, G\right)=\inf \left\{d\left(z_{1}, z_{2}\right): z_{2} \in G\right\}$. For $r>0$ we denote by $S(r)$ the set $\left\{x \in R^{n}:|x|<r\right\}$. Let $H$ be a nonempty, proper subset of $R^{n}$ such that $H \subset S(r), \bar{H}$ its closure and fr $H$ its boundary. For any two positive 
numbers $r_{1}$ and $r_{2}, r_{1}<r_{2}$, we write

$$
\begin{aligned}
S\left(H, r_{1}\right) & =\left\{x \in S(r): d(x, H)<r_{1}\right\}, \\
S\left[H, r_{1}\right] & =\left\{x \in S(r): d(x, H) \leq r_{1}\right\}, \\
S^{c}\left[H, r_{1}\right] & =\left\{x \in S(r): x \notin S\left[H, r_{1}\right]\right\}, \\
A\left(H, r_{1}, r_{2}\right) & =\left\{x \in S(r): r_{1}<d(x, H)<r_{2}\right\}, \\
\Delta\left(H, r_{1}, r_{2}\right) & =\left\{x \in S(r): x \in S\left(r_{2}\right) \text { and } x \in S^{c}\left[H, r_{1}\right] .\right.
\end{aligned}
$$

DEFINITION 1. A real-valued function $\psi(\cdot)$ belongs to the class $K$ if $\psi(\cdot)$ is continuous and strictly increasing on $[0, r)$ and $\psi(0)=0$.

Consider a function $F: D_{r} \rightarrow R$ and, for a given $t \geq 0$, denote by $E_{t}(F=0)$ the set of points $x \in S(r)$ for which $F(t, x)=0$. If $F$ is independent of $t$, all the sets $E_{t}(F=0)$ are equal to one and the same set which we denote by $E(F=0)$.

DEFINITION 2. The function $F$ is positive definite with respect to the set $H$ if $F(t, x)=0$ for all $(t, x) \in[0, \infty) \times H$ and there exists a function $\psi \in K$ such that $F(t, x) \geq \psi(d(x, H))$ for all $t \geq 0$ and $x \in S(r) \backslash H$.

DEFINITION 3. A function $\Phi: D_{r} \rightarrow R$ is definitely nonzero on the set $E_{t}(F=0) \backslash \bar{H}$ (respectively on the set $E(F=0) \backslash \bar{H}$ ) if for any two positive numbers $\varepsilon_{1}$ and $\varepsilon_{2}, \varepsilon_{1}<\varepsilon_{2}<r$, there exist positive numbers $\delta_{1}=\delta_{1}\left(\varepsilon_{1}, \varepsilon_{2}\right)$ and $\delta_{2}=\delta_{2}\left(\varepsilon_{1}, \varepsilon_{2}\right)$ such that $|\Phi(t, x)| \geq \delta_{2}$ for all $t \geq 0$ whenever $x \in \Delta\left(H, \varepsilon_{1}, \varepsilon_{2}\right)$ and $d\left(x, E_{t}(F=0)\right)<\delta_{1}$ (respectively $\left.d(x, E(F=0))<\delta_{1}\right)$.

DEFINITION 4. A function $\Phi: D_{r} \rightarrow R$ has an infinitesimal upper bound on the set $E_{t}(F=0) \backslash \bar{H}$ (respectively on the set $E(F=0) \backslash \bar{H}$ ) if $\Phi(t, x)=0$ for all $t \geq 0$ and $x \in E_{t}(F=0) \backslash \bar{H}$ (respectively $x \in E(F=0) \backslash \bar{H}$ ) and for any positive numbers $\varepsilon_{1}, \varepsilon_{2}$ and $\rho, \varepsilon_{1}<\varepsilon_{2}<r$, there exists a positive number $\delta=\delta\left(\varepsilon_{1}, \varepsilon_{2}, \rho\right)$ such that $|\Phi(t, x)| \leq \rho$ for all $t \geq 0$ whenever $x \in \Delta\left(H, \varepsilon_{1}, \varepsilon_{2}\right)$ and $d\left(x, E_{t}(F=0)\right)<\delta$ (respectively $d(x, E(F=0))<\delta)$.

DEFINITION 5. The set $H$ is

(i) totally stable or stable under persistent disturbances with respect to $(\mathrm{N})$ if for every $\varepsilon>0$ and every $t_{0} \geq 0$ two positive numbers $\eta_{1}=\eta_{1}\left(\varepsilon, t_{0}\right)$ and $\eta_{2}=\eta_{2}\left(\varepsilon, t_{0}\right)$ can be found such that $d\left(H, y\left(t, t_{0}, y_{0}\right)\right)<\varepsilon$ for every solution $y\left(t, t_{0}, y_{0}\right)$ of $(\mathrm{P})$ and for all $t \geq t_{0}$ whenever $y_{0} \in S(r), d\left(H, y_{0}\right) \leq \eta_{1}$ and $|g(t, y)| \leq \eta_{2}$ for all $t \geq t_{0}$ and $y \in \overline{S(H, \varepsilon)}$;

(ii) uniformly totally stable with respect to $(\mathrm{N})$ if (i) holds with $\eta_{1}=\eta_{1}(\varepsilon)$ and $\eta_{2}=\eta_{2}(\varepsilon)$.

Definition 1 is due to Hahn $[\mathbf{1 0}$, p. 7]. If $H=\{0\}$, then Definition 2 reduces to the standard definition of positive definiteness $[10$, p. 4] while Definitions 3 and 4 reduce to Definitions 1.1 and 1.5, respectively, of Matrosov [3]. If, in addition, $f(t, 0)=0$ for all $t \geq 0$, then Definition 5(i) reduces to Duboshin's definition of total stability of the zero solution of $(\mathrm{N})$. We note that in the definition of total stability, $\eta_{1}$ and $\eta_{2}$ may be replaced by a single $\eta=\eta_{1}=\eta_{2}$.

Let $v(t, x)$ be a continuous real-valued functions on $D_{r}$. The generalized total derivative of $v$ with respect to $(\mathrm{N})$ is given by

$$
\dot{v}_{(\mathrm{N})}(t, x)=\limsup _{h \rightarrow 0^{+}} h^{-1}[v(t+h, x+h f(t, x))-v(t, x)] .
$$

If $v$ is continuously differentiable on $D_{r}$, then

$$
\dot{v}_{(\mathrm{N})}(t, x)=\partial v(t, x) / \partial t+\operatorname{grad} v(t, x) \cdot f(t, x)
$$


and

$$
\dot{v}_{(\mathrm{P})}(t, x)=\dot{v}_{(\mathrm{N})}(t, x)+\operatorname{grad} v(t, x) \cdot g(t, x),
$$

where $\operatorname{grad} v=\left(\partial v / \partial x_{1}, \ldots, \partial v / \partial x_{n}\right)$ and - denotes the inner product of two vectors.

3. Main results. We now present the results pertaining to the total stability of the set $H$ with respect to $(\mathrm{N})$.

THEOREM 3.1. Let $f(t, x)$ be bounded on $D_{r}$ for $(\mathrm{N})$ and let $H$ be a nonempty proper subset of $R^{n}$ such that $S\left(H, r_{1}\right) \subset S(r)$. Suppose that there exist two realvalued functions $U(t, x)$ and $V(t, x)$ defined, continuous and continuously differentiable on $D_{r}$ and such that

(a) $U(t, x)$ is positive definite with respect to $H$;

(b) $U_{(\mathrm{N})}(t, x) \leq-W(x) \leq 0$, where $W$ is a real-valued function defined and continuous on $S(r)$;

(c) There are two positive numbers $m_{1}$ and $m_{2}$ such that $|\operatorname{grad} U(t, x)| \leq m_{1}$ and $|\operatorname{grad} V(t, x)| \leq m_{2}$ on $D_{r}$

(d) $V(t, x)$ has an infinitesimal upper bound on the set $E(W=0) / \overline{H \cap E(W=0)}$;

(e) $\dot{V}_{(\mathrm{N})}(t, x)$ is definitely nonzero on the set $E(W=0) \backslash \overline{H \cap E(W=0)}$.

Then the set $H$ is uniformly totally stable with respect to $(\mathrm{N})$.

Proof. Let $M$ be a bound for $f(t, x),|f(t, x)|<M$, for $(t, x) \in D_{r}$. Since $\bar{H} \subset S(r)$ there is a positive number $\rho, \rho<r$, such that $|x| \leq \rho$ for all $x \in H$. Let a positive number $\varepsilon<r-\rho$ be given. For this $\varepsilon$ and $t_{0} \geq 0$ we define

$$
2 m=\inf \left\{U(t, x): t \geq t_{0}, d(x, H)=\varepsilon\right\} .
$$

From (a) it follows that $m>0$. Pick positive numbers $\varepsilon_{1}=\varepsilon_{1}\left(t_{0}, \varepsilon\right)$ and $\varepsilon_{2}=$ $\varepsilon_{2}\left(t_{0}, \varepsilon\right), \varepsilon_{1}<\varepsilon_{2}<\varepsilon$, and define

$$
\begin{aligned}
k_{1} & =\sup \left\{U(t, x): t \geq t_{0}, x \in S\left[H, \varepsilon_{1}\right]\right\}, \\
k_{2} & =\sup \left\{U(t, x): t \geq t_{0}, x \in S\left[H, \varepsilon_{2}\right]\right\}, \\
l & =\inf \left\{U(t, x): t \geq t_{0}, x \in A\left(H, \varepsilon_{2}, \varepsilon\right)\right\} .
\end{aligned}
$$

Using conditions (a) and (c) we may pick $\varepsilon_{1}$ and $\varepsilon_{2}$ so that $k_{2}<m$ and $k_{1}<l$. Further, using condition (e), choose positive numbers $\delta_{1}=\delta_{1}\left(\varepsilon_{1}, \varepsilon_{2}\right)$ and $\delta_{2}=$ $\delta_{2}\left(\varepsilon_{1}, \varepsilon_{2}\right), \delta_{1} \leq \varepsilon_{1}$, such that $\left|\dot{V}_{(\mathrm{N})}(t, y)\right| \geq \delta_{2}$ for all $t \geq t_{0}$ whenever $y \in A\left(H, \varepsilon_{1}, \varepsilon\right)$ and $d(y, E(W=0))<\delta_{1}$. By virtue of (b) we have

$$
J=\left\{\inf W(x): x \in A\left(H, \varepsilon_{1}, \varepsilon\right), d(x, E(W=0)) \geq \delta_{0} / 2\right\}>0
$$

for any positive number $\delta_{0}<\delta_{1}$.

For $t \geq t_{0} \geq 0$ and $y_{0} \in H$ we consider a solution $y(t)=y\left(t, t_{0}, y_{0}\right)$ of $(\mathrm{P})$ and establish some of its properties. First we obtain an upper bound for the escape time of $y(t)$ from a compact set.

1. If $|g(t, y)| \leq \delta_{2} / 2 m_{2} n$ for $t \geq t_{0}$ and $y \in \overline{S(H, \varepsilon)}$, then $y(t)$ cannot remain from a certain $t \geq t_{0}$ onward in the set

$$
B=\left\{y: y \in A\left(H, \varepsilon_{1}, \varepsilon\right), d(y, E(W=0))<\delta_{0}\right\} .
$$

From (c) and (d) and by the Mean Value Theorem it follows that $|V(t, y)|<$ $m_{2} n \delta_{0}$ for all $t \geq t_{0}$ and $y \in B$. Let us suppose that $y(t) \in B$ for every $t$ in some 
closed interval $\left[t_{1}, t_{2}\right], t_{1} \geq t_{0}$. If we write $V(t)$ for $V(t, y(t))$ and $\dot{V}(t)$ for $\dot{V}(t, y(t))$, then

$$
V\left(t_{2}\right)-V\left(t_{1}\right)=\int_{t_{1}}^{t_{2}} \dot{V}_{(\mathrm{P})}(t) d t
$$

Now for as long as $y(t)$ exists,

$$
\dot{V}_{(\mathrm{P})}(t)=\dot{V}_{(\mathrm{N})}(t)+\operatorname{grad} V(t) \cdot g(t, y(t)),
$$

which by using condition (c) and since $\left|\dot{V}_{(\mathrm{N})}(t, y)\right| \geq \delta_{2}$ and $|g(t, y)| \leq \delta_{2} / 2 m_{2} n$ yields

$$
\left|\dot{V}_{(\mathrm{P})}(t)\right| \geq\left|\dot{V}_{(\mathrm{N})}(t)\right|-|\operatorname{grad} V(t) \cdot g(t, y(t))| \geq \delta_{2}-\delta_{2} / 2 n \geq \delta_{2} / 2
$$

for $t \in\left[t_{1}, t_{2}\right]$. Thus from (2), one gets successively

$$
\begin{aligned}
2 m_{2} n \delta_{0} & >\left|V\left(t_{2}\right)\right|+\left|V\left(t_{1}\right)\right| \geq\left|V\left(t_{2}\right)-V\left(t_{1}\right)\right| \\
& =\left|\int_{t_{1}}^{t_{2}} \dot{V}_{(\mathrm{P})}(t) d t\right|=\int_{t_{1}}^{t_{2}}\left|\dot{V}_{(\mathrm{P})}(t)\right| d t \\
& \geq \delta_{2}\left(t_{2}-t_{1}\right) / 2
\end{aligned}
$$

which yields $t_{2}-t_{1}<4 m_{2} n \delta_{0} / \delta_{2}$. Hence, there exists $t, t_{1}<t \leq t_{1}+4 m_{2} n \delta_{0} / \delta_{2}$, such that $y(t) \in$ fr $B$ and this proves the assertion.

Note that we are able to write that the absolute value of the integral is equal to the integral of the absolute value because $V$ is continuous and therefore does not change sign on $\left[t_{1}, t_{2}\right]$.

2. If $|g(t, y)| \leq J / c m_{1} n$ on $\left[t_{0}, \infty\right) \times \overline{(H, \varepsilon)}$, where $c$ is a positive constant to be determined later, then $y(t)$ cannot remain in the set

$$
B_{1}=\left\{y: y \in A\left(H, \varepsilon_{1}, \varepsilon\right), d(y, E(W=0)) \geq \delta_{0} / 2\right\} \text {. }
$$

In fact, suppose that $y(t) \in B_{1}$ for $t \geq \tau>t_{0}$. We write $U(t)$ for $U(t, y(t))$ and $\dot{U}(t)$ for $U(t, y(t))$. Then

$$
U(t)-U(\tau)=\int_{\tau}^{t} \dot{U}_{(\mathrm{P})}(s) d s .
$$

From

$$
\dot{U}_{(\mathrm{P})}(t)=\dot{U}_{(\mathrm{N})}(t)+\operatorname{grad} U(t) \cdot g(t, y(t)),
$$

by using (1) and conditions (b) and (c) one has

$$
\dot{U}_{(\mathrm{P})}(t) \leq-J+m_{1} n\left(J / c m_{1} n\right)=(1-c) J / c
$$

and thus

$$
U(t) \leq U(\tau)+(1-c) J(t-\tau) / c .
$$

Choose $c>1$. Then if $t$ is sufficiently large we would have $U(t)<0$. This contradicts condition (a) and thereby proves our assertion.

We now obtain a lower bound for the transit time of $y(t)$.

3. Suppose there are $t_{1}$ and $t_{2}, t_{0} \leq t_{1}<t_{2}$. If $y(t)$ exists on $\left[t_{1}, t_{2}\right]$ and if $|g(t, y)| \leq \eta_{2}$ for all $t \geq t_{0}$ and $y \in \overline{S(H, \varepsilon)}$, where $\eta_{2}=\min \left(\delta_{2} / 2 m_{2} n, J / c m_{1} n\right)$, then $d\left(y\left(t_{1}\right), y\left(t_{2}\right)\right) \geq p>0$ implies that $t_{2}-t_{1} \geq p /\left(M+\eta_{2}\right) n^{1 / 2}$. 
By the Mean Value Theorems we have

$$
\left|y_{i}\left(t_{2}\right)-y_{i}\left(t_{1}\right)\right|=\left|d y_{i}\left(\tau_{i}\right) / d t\right|\left(t_{2}-t_{1}\right), \quad i=1,2, \ldots, n,
$$

where $t_{i} \leq \tau_{i} \leq t_{2}$. But

$$
\left|d y_{i}\left(\tau_{i}\right) / d t\right|=\left|f_{i}\left(\tau_{i}, y\left(\tau_{i}\right)\right)+g_{i}\left(\tau_{i}, y\left(\tau_{i}\right)\right)\right| \leq M+\eta_{2}
$$

and thus $\left|y_{i}\left(t_{2}\right)-y_{i}\left(t_{1}\right)\right| \leq\left(M+\eta_{2}\right)\left(t_{2}-t_{1}\right)$. Hence, by summation, the desired result follows.

4. If, for some $\tau_{0}>t_{0}, y\left(\tau_{0}\right) \in A\left(H, \varepsilon_{1}, \varepsilon\right)$ and $d\left(y\left(\tau_{0}\right), E(W=0)\right) \leq \delta_{0} / 2$ and if $y(t) \in A\left(H, \varepsilon_{1}, \varepsilon\right)$ for each $t$ with $\tau_{0} \leq t \leq \tau_{0}+4 m_{2} n_{0} / \delta_{2}$, then $y\left(\tau_{1}\right) \in A\left(H, \varepsilon_{1}, \varepsilon\right)$ and $d\left(y\left(\tau_{1}\right), E(W=0)\right)=\delta_{0}$ imply $U\left(\tau_{1}\right) \leq U\left(\tau_{0}\right)-J \delta_{0} / 2 c\left(M+\eta_{2}\right) n^{-1 / 2}$ on $\tau_{0} \leq \tau_{1} \leq \tau_{0}+4 m_{2} n \delta_{0} / \delta_{2}$ for $c \geq 2+8 m_{2} n^{3 / 2}\left(M+\eta_{2}\right) / \delta_{2}$.

From the imposed conditions it follows that there is $\tau^{*}, \tau_{0}<\tau^{*}<\tau_{1}$, such that $y\left(\tau^{*}\right) \in A\left(H, \varepsilon_{1}, \varepsilon\right)$ and $d\left(y\left(\tau^{*}\right), E(W=0)\right)=\delta_{0} / 2$, and $y(t) \in A\left(H, \varepsilon_{1}, \varepsilon\right)$ and $\delta_{0} / 2 \leq d(y(t), E(W=0)) \leq \delta_{0}$ for all $t$ with $\tau^{*} \leq t \leq \tau_{1}$. But $d\left(y\left(\tau^{*}\right), y\left(\tau_{1}\right)\right) \geq \delta_{0} / 2$ and then, by using the proved properties 1 and 3 of $y(t)$, we obtain

$$
4 m_{2} n \delta_{0} / \delta_{2} \geq \tau_{1}-\tau^{*} \geq \delta_{0} / 2\left(M+\eta_{2}\right) n^{1 / 2}
$$

On the other hand, from (3) and by conditions (b) and (c) of the theorem, it follows that $\dot{U}_{(\mathrm{P})}(t) \leq m_{1} n \eta_{2}$ on $\tau_{0} \leq t \leq \tau^{*}$ while from (4) we have $\dot{U}_{(\mathrm{P})}(t) \leq(1-c) \mathrm{J} / \mathrm{c}$ on $\tau^{*} \leq t \leq \tau_{1}$. Thus

$$
\begin{aligned}
U\left(\tau_{1}\right)-U\left(\tau_{0}\right) & =\int_{\tau_{0}}^{\tau^{*}} \dot{U}_{(\mathrm{P})}(t) d t+\int_{\tau^{*}}^{\tau_{1}} \dot{U}_{(\mathrm{P})}(t) d t \\
& \leq 4 m_{1} m_{2} n^{2} \eta_{2} \delta_{0} / \delta_{2}+(1-c) J \delta_{0} / 2 c\left(M+\eta_{2}\right) n^{1 / 2}
\end{aligned}
$$

and, by the definition of $\eta_{2}$ in 3 , this yields

$$
U\left(\tau_{1}\right) \leq U\left(\tau_{0}\right)+(J / c)\left(4 m_{2} n \delta_{0} / \delta_{2}\right)+(1-c) \delta_{0} / 2\left(M+\eta_{2}\right) n^{1 / 2} .
$$

Taking

$$
c \geq 2+8 m_{2} n^{3 / 2}\left(M+\eta_{2}\right) / \delta_{2}
$$

we obtain $U\left(\tau_{1}\right) \leq U\left(\tau_{0}\right)-J \delta_{0} / 2 c\left(M+\eta_{2}\right) n^{1 / 2}$ on $\tau_{0} \leq \tau_{1} \leq \tau_{0}+4 m_{2} n \delta_{0} / \delta_{2}$ and the assertion is proved.

From now on we suppose that (5) holds.

5. If, for some $t^{*}>t_{0}, \varepsilon_{1} \leq d\left(y\left(t^{*}\right), H\right) \leq \varepsilon_{2}$ and if $|g(t, y)| \leq \eta_{2}$ for all $t \geq$ $t_{0}, y \in \overline{S(H, \varepsilon)}$ and with $\eta_{2}$ as in 3 , then $y(t) \in S(H, \varepsilon)$ on $t^{*} \leq t \leq t^{*}+4 m_{2} n \delta_{0} / \delta_{2}$.

From (3) and by using (1) and conditions (b) and (c) of the theorem we have $\dot{U}_{(\mathrm{P})}(t) \leq J / c$ for all $t>t^{*}$ for which $y(t) \in S(H, \varepsilon)$. Integrating on $\left[t^{*}, t\right]$ we obtain $U(t) \leq \bar{U}\left(t^{*}\right)+(J / c)\left(t-t^{*}\right)$ which by property 1 and the definition of $m$ yields

$$
U(t) \leq m+(J / c) 4 m_{2} n \delta_{0} / \delta_{2} \text {. }
$$

From (1) we see that $J \rightarrow 0$ as $\delta_{0} \rightarrow 0$. Then we may pick $\delta_{0}$ small enough so that $J \delta_{0} / 2 M n^{1 / 2}<m$. Thus (6) becomes

$$
U(t) \leq m+\left(J \delta_{0}\right) / 2 n^{1 / 2}\left(M+\eta_{2}\right)<2 m
$$

and this implies that $y(t) \in S(H, \varepsilon)$ on $t^{*} \leq t \leq t^{*}+4 m_{2} n \delta_{0} / \delta_{2}$, proving the assertion. 
We shall now use properties 1-5 of $y(t)$ in order to complete the proof. We have to prove that any solution $y(t)$ of $(\mathrm{P})$ with $y\left(t_{0}\right)=y_{0}$ satisfies $d(y(t), H)<\varepsilon$ for all $t \geq t_{0}$ whenever $y_{0} \in S\left(H, \varepsilon_{1}\right)$ and $|g(t, y)| \leq \eta_{2}$ for all $t \geq t_{0}$ and $y \in \overline{S(H, \varepsilon)}$, where $\eta_{2}$ is defined in 3 .

Let us argue by contradiction, and thus suppose that there exists a $T>t_{0}$ such that $d(y(T), H)=\varepsilon$. Since $y(t)$ is continuous, there exist $t_{1}, t_{0}<t_{1}<T$, and $t_{2}, t_{1}<t_{2}<T$, with the properties that $d\left(y\left(t_{1}\right), H\right)=\varepsilon_{1}, d\left(y\left(t_{2}\right), H\right)=$ $\varepsilon_{2}, y(t) \in A\left(H, \varepsilon_{1}, \varepsilon\right)$ on $t_{1}<t<T$ and $y(t) \in A\left(H, \varepsilon_{2}, \varepsilon\right)$ on $t_{2}<t<T$. Let $y(t), t_{1}<t \leq t_{2}$, belong to the set $B_{1}$ defined in 2 . Arguing now as in 2 we obtain

$$
U(t)-U\left(t_{1}\right) \leq(1-c) J\left(t-t_{1}\right) / c
$$

which by the definitions of $k_{1}$ and $l$ yields for $t=t_{2}$

$$
l \leq U\left(t_{2}\right) \leq k_{1}+(1-c) J\left(t-t_{1}\right) / c<k_{1}
$$

But $\varepsilon_{1}$ and $\varepsilon_{2}$ have been chosen so that $k_{1}<l$. From the obtained contradiction it follows that there is $s_{1}, t_{1} \leq s_{1}<t_{2}$, such that $\varepsilon_{1} \leq d\left(y\left(s_{1}\right), H\right)<$ $\varepsilon_{2}, d\left(y\left(s_{1}\right), E(W=0)\right)=\delta_{0} / 2$ and $d(y(t), E(W=0))<\delta_{0} / 2$ in a right neighborhood of $s_{1}$. Property 5 implies that $y(t) \in S(H, \varepsilon)$ for $s_{1} \leq t \leq s_{1}+4 m_{2} n \delta_{0} / \delta_{2}$, and from property 1 it follows that there exists $\tau_{1}, s_{1}<\tau_{1} \leq s_{1}+4 m_{2} n \delta_{0} / \delta_{2}$, such that $d\left(y\left(\tau_{1}\right), E(W=0)\right)=\delta_{0}$. For this $\tau_{1}$ and by property 4 we have

$$
U\left(\tau_{1}\right) \leq U\left(s_{1}\right)-J \delta_{0} / 2 c\left(M+\eta_{2}\right) n^{1 / 2}
$$

and (7) with $t$ replaced by $s_{1}$ and (8) readily imply

$$
U\left(\tau_{1}\right) \leq U\left(t_{1}\right)+(1-c) J\left(s_{1}-t_{1}\right) / c-J \delta_{0} / 2 c\left(M+\eta_{2}\right) n^{1 / 2} .
$$

Then, using the definition of $k_{1}$, we obtain $U\left(\tau_{1}\right)<k_{1}$ and hence, $y\left(\tau_{1}\right) \in$ $A\left(H, \varepsilon_{1}, \varepsilon_{2}\right)$ for $\tau_{1}<t_{2}$. Proceeding in the same manner for $t>\tau_{1}$ we see that on the interval $\left(\tau_{1}, t_{2}\right)$ there are $s_{2}$ and $\tau_{2}, s_{2}<\tau_{2}$, such that $d\left(y\left(\tau_{2}\right), y\left(s_{2}\right)\right) \geq \delta_{0} / 2$. By property 3 we obtain $\tau_{2}-s_{2} \geq \delta_{0} / 2\left(M+\eta_{2}\right) n^{1 / 2}$. Then from (7) with $t$ and $t_{1}$ replaced by $\tau_{2}$ and $s_{2}$, respectively, and by property 4 it follows that

$$
U\left(\tau_{2}\right) \leq U\left(s_{2}\right)-(1+c) J \delta_{0} / 2 c\left(M+\eta_{2}\right) n^{1 / 2} .
$$

Hence, $U\left(\tau_{2}\right)<k_{1}$ and thus $y\left(\tau_{2}\right) \in A\left(H, \varepsilon_{1}, \varepsilon_{2}\right)$ for $\tau_{2}<t_{2}$. Continuing in this fashion, two increasing sequences $\left\{s_{j}\right\}$ and $\left\{\tau_{j}\right\}$ with $t_{1} \leq s_{j}<\tau_{j}<t_{2}, j=1,2, \ldots$, are obtained. Since $\tau_{j}-s_{j} \geq \delta_{0} / 2\left(M+\eta_{2}\right) n^{1 / 2}, j=1,2, \ldots$, it follows that the sequence of disjoint intervals $\left\{\left(s_{j}, \tau_{j}\right)\right\}$ is unbounded. Therefore a finite $t_{2}$ and consequently a finite $T>t_{2}$ such that $d(y(T), H)=k$ does not exist. This contradicts our assumption and proves the theorem.

Some remarks concerning the above theorem are in order here. First it is not difficult to show that Theorem 3.1 holds if condition (d) is dropped; obviously, in this case, the definition of $\eta_{2}$ in 3 and (5) must be slightly changed. Further, if $H=\{0\}$ and $f(t, 0)=0$ for all $t \geq 0$, then the celebrated theorem of Malkin [20] and Gorshin [21] on the total stability of the zero solution for $(\mathrm{N})$ can be treated as a corollary to our result. Even in this special case, Theorem 3.1 improves their result. In particular, this traditional result requires the derivative of a Liapunov function along the solutions of $(\mathrm{N})$ to be negative definite. In Theorem 3.1 this requirement is relaxed considerably. With the introduction of the auxiliary function 
$W(x)$ one estimates, so to speak, the vanishing of the derivative $\dot{U}_{(\mathrm{N})}(t, x)$. Finally, the hypotheses of Theorem 3.1 do not imply that any solution $y\left(t, t_{0}, y_{0}\right)$ of $(\mathrm{P})$ tends to $H$ as $t \rightarrow \infty$. In fact, $g(t, y)$ does not vanish, nor does it diminish as $t \rightarrow \infty$. However, some kind of asymptotic property can be proved. Namely, in the following theorem, we show that if $g$ in (P) is sufficiently small, then the solutions of $(\mathrm{P})$ starting in the vicinity of $H$ are rejected and remain in an arbitrarily small neighborhood of $H$ although they do not tend to $H$ asymptotically. More precisely, we have the following result.

THEOREM 3.2. Let the conditions of Theorem 3.1 be satisfied. For every $\varepsilon>0$ there is an $\eta_{1}>0$ and for some $\delta>0$ there is an $\eta_{2}^{*}>0$ such that if $t_{0} \geq 0, y_{0} \in$ $S\left(H, \eta_{1}\right)$ and if $|g(t, y)| \leq \eta_{2}^{*}$ for all $y \in S(H, \varepsilon)$ and all $t \geq t_{0}$, then there exists $a T^{*}>0$ such that the solution $y\left(t, t_{0}, y_{0}\right)$ of $(\mathrm{P})$ satisfies $d\left(y\left(t, t_{0}, y_{0}\right), H\right)<\delta$ for all $t \geq T^{*}$.

ProOF. For every $t_{0} \geq 0$ and every $\varepsilon>0$ we pick $\eta_{1}$ and $\eta_{2}$ as in the definition of total stability of the set $H$. Thus any solution $y(t)=y\left(t, t_{0}, y_{0}\right)$ of $(\mathrm{P})$ starting in $s\left(H, \eta_{1}\right)$ remains in $S(H, \varepsilon)$. For a given $\delta, 0<\delta<\eta_{1}$, let $\eta_{1}^{*}$ and $\eta_{2}^{*}<\eta_{2}$ be chosen as in the definition of total stability of $H$ with $\varepsilon$ repalced by $\delta$. Then, for $y \in A\left(H, \eta_{1}^{*}, \varepsilon\right)$ and if $|g(t, y)|<\eta_{2}^{*}$ on $\left[t_{0}, \infty\right) \times S(H, \varepsilon)$, we obtain $\dot{U}_{(\mathrm{P})}(t, y)<0$. Therefore, for $y_{0} \in S\left(H, \eta_{1}\right), y(t)$ enters at least once inside $S\left(H, \eta_{1}^{*}\right)$ and remains thereafter in $S(H, \delta)$ because of total stability of $H$. In fact, from Theorem 3.1 we have $d(y(t), H)<\varepsilon$ for all $t \geq t_{0}$ whenever $y_{0} \in S\left(H, \eta_{1}^{*}\right)$ and $|g(t, y)| \leq \eta_{2}^{*}$ on $\left[t_{0}, \infty\right) \times \overline{S(H, \varepsilon)}$. Suppose that $y(t) \in A(H, \delta, \varepsilon)$ for all $t>t_{1}$, where $t_{1}>t_{0}$ is such that $d\left(y\left(t_{1}\right), H\right)=\delta$. Then, by induction, from (9) and (10) it follows that

$$
U\left(\tau_{j}\right) \leq k_{1}-(1+c(j-1)) J \delta_{0} / 2 c\left(M+\eta_{2}\right) n^{1 / 2}, \quad j=1,2, \ldots,
$$

and $U\left(\tau_{j}\right) \rightarrow-\infty$ as $j \rightarrow \infty$. This contradicts condition (a) of Theorem 3.1. Thus there exists a $T^{*}>0$ such that $d(y(t), H)<\delta$ for all $t \geq T^{*}$ and the proof is complete.

In Theorem 3.1, condition (b) can be preserved in the form $\dot{U}_{(\mathrm{N})}(t, x) \leq 0$ if the first and second partial derivatives of $U(t, x)$ are bounded on $D_{r}$, and conditions (d) and (e) can be produced on the set $E_{t}(\dot{U}=0) \backslash \overline{H \cap E_{t}(\dot{U}=0)}$. Then we arrive at the following modification of Theorem 3.1.

THEOREM 3.3. Let $f(t, x)$ be bounded on $D_{r}$ for $(\mathrm{N})$ and let $H$ be a nonempty proper subset of $R^{n}$ such that $S\left(H, r_{1}\right) \subset S(r)$. Suppose that there exist two realvalued functions $U(t, x)$ and $V(t, x)$ defined, continuous and continuously differentiable on $D_{r}$ and such that

(a) $U(t, x)$ is positive definite with respect to $H$;

(b) $\dot{U}_{(\mathrm{N})}(t, x) \leq 0$ and the first and second partial derivatives of $U(t, x)$ are continuous and bounded on $D_{r}$;

(c) There is a positive number $m_{2}$ such that $|\operatorname{grad} V(t, x)| \leq m_{2}$ on $D_{r}$;

(d) For any $t \geq 0, V(t, x)$ has an infinitesimal upper bound on the set $E_{t}(\dot{U}=0) \backslash \overline{H \cap E_{t}(\dot{U}=0)}$;

(e) For any $t \geq 0, \dot{V}_{(\mathrm{N})}(t, x)$ is definitely nonzero on the set

$$
E_{t}(\dot{U}=0) \backslash \overline{H \cap E_{t}(\dot{U}=0)} .
$$

Then the set $H$ is totally stable with respect to $(\mathrm{N})$. 
The proof of this theorem follows the same line of reasoning as the proof of Theorem 3.1, and is thus omitted.

4. Applications. Let a natural system be governed by an $n$-vector $x$ subject to a differential equation of the form

$$
\dot{x}=A x,
$$

where $A$ is an $n \times n$ constant matrix. This equation is usually called the plant equation. The practical problem is to restore the system as strongly as possible to the origin $x=0$, whenever it undergoes any small deviation. This is often done by means of an additional mechanism operating through a scalar parameter $\xi$. The effect of the deviation on $\xi$ is by means of a "signal" $\sigma$ depending linearly on $x$ and $\xi$. The new system assumed the form

$$
\dot{x}=A x+\xi b, \quad \sigma=c^{\prime} x-\rho \xi,
$$

where $b$ and $c$ are $n$-vectors, $\rho>0$ is a scalar and the prime denotes transpose. The two principal types discussed by many authors are: (I) Indirect control: $\dot{\xi}=\varphi(\sigma)$ and (II) Direct control: $\xi=\varphi(\sigma), \sigma=c^{\prime} x(\rho=0)$. The scalar function $\varphi(\sigma)$ is the characteristic of the control. One assumes that $\varphi(\sigma)$ is continuous for all $\sigma$ and behaves so as to guarantee existence and uniqueness of the solutions of the differential systems in which it occurs and, moreover, $\sigma \varphi(\sigma)>0$ for $\sigma \neq 0$. Denote by $F$ the class of all such functions.

The basic problem posed by Lurie is to find conditions on $b, c$, and $A$ under which the system (12) is absolutely stable, i.e., that the origin is asymptotically stable in the large for every $\varphi \in F$. Though this problem is similar to the one in the study of total stability of an equilibrium point considered by Malkin and Gorshin, it differs from it in one important aspect: the perturbations need not be uniformly bounded. Indeed, since they are allowed to be linear in $\sigma$, the problem is essentially an algebraic one. As a general reference to the Lurie problem, and for a good bibliography, we can refer to the book of Lefschetz [22]. The reader may find a complete presentation of the original results as well as of the historical background of this problem in the monograph of Aizerman and Gantmakher [23].

Consider the system

$$
\dot{x}=A(t) x,
$$

where $x$ is an $n$-vector and $A(t)$ is a continuous $n \times n$ matrix on $[0, \infty)$. As is well known in stability theory the eigenvalues of the matrix $A(t)$ cannot be used to determine the asymptotic behavior of the solutions of (13). We suppose that the system (13) is uniformly asymptotically stable. Then there are positive constants $L$ and $\alpha$ such that the solution $x\left(t, t_{0}, x_{0}\right)$ of $(13)$ with $x\left(t_{0}, t_{0}, x_{0}\right)=x_{0}$ satisfies

$$
\left|x\left(t, t_{0}, x_{0}\right)\right| \leq L\left|x_{0}\right| e^{-\alpha\left(t-t_{0}\right)}
$$

for all $t \geq t_{0} \geq 0$ and all $x_{0}$ in $R^{n}$. By a theorem of Malkin [24] and Massera [25] it follows that there exists a continuous scalar function $V$ on $[0, \infty) \times R^{n}$ such that

(i) $|x| \leq V(t, x) \leq L|x|$,

(ii) $\dot{V}_{(13)}(t, x) \leq-\alpha V(t, x)$,

(iii) $|V(t, x)-V(t, y)| \leq L|x-y|$ 
for all $t \geq 0$ and $x, y$ in $R^{n}$. Notice that the linear system (13) is uniformly asymptotically stable if and only if it is exponentially stable [25].

Let us first consider the nonautonomous direct control system

$$
\dot{x}=A(t) x+b \varphi(\sigma), \quad \sigma=c^{\prime} x,
$$

and its perturbed system

$$
\dot{x}=A(t) x+b \varphi(\sigma)+g(t, x), \quad \sigma=c^{\prime} x,
$$

where the function $g(t, x)$ is defined and continuous on $[0, \infty) \times R^{n}$ and has the meaning of a constantly acting perturbation and the rest of the letters have the same meaning as above. The following theorem holds.

THEOREM 4.1. Let $|A(t)| \leq A$ uniformly on $[0, \infty), \varphi \in F, c^{\prime} b=-d<$ 0 , and let the system (13) be uniformly asymptotically stable. Then, if $4 \alpha d>$ $\left(L^{2}|b|+A|c|\right)^{2}$, the zero solution of $(16)$ is totally stable.

Proof. Consider the function $\Phi(x)=\int_{0}^{\sigma} \varphi(s) d s, \sigma=c^{\prime} x$. Its derivative along the solutions of $(16)$ is

$$
\dot{\Phi}_{(16)}(x)=c^{\prime} A(t) x \varphi(\sigma)-d \varphi^{2}(\sigma) .
$$

Since the zero solution of (13) is uniformly asymptotically stable there is a continuous scalar function $V(t, x)$ satisfying (15). The derivative of $V(t, x)$ along the solutions of (16) satisfies

$$
\dot{V}_{(16)}(t, x) \leq \dot{V}_{(13)}(t, x)+L|b||\varphi(\sigma)|
$$

which because of properties (i) and (ii) in (15) becomes

$$
\dot{V}_{(16)}(t, x) \leq-\alpha|x|+L|b||\varphi(\sigma)| \text {. }
$$

Define the function $U(t, x)=V^{2}(t, x) / 2+\Phi(x)$. It is easy to see that $U(t, x)$ is positive definite, radially unbounded and has an infinitesimal upper bound since $V(t, x)$ has all these properties. Taking the derivative of $U(t, x)$ along the solutions of (16) we obtain

$$
\dot{U}_{(16)}(t, x) \leq-\alpha|x|^{2}+|x|\left(L^{2}|b|+A|c|\right)|\varphi(\sigma)|-d \varphi^{2}(\sigma) .
$$

Here we have used (18) and (20). Let us consider the right-hand side of this inequality as a quadratic form in $|x|$ and $|\varphi(\sigma)|$. The condition for it to be negative definite is $4 \alpha d>\left(L^{2}|b|+A|c|\right)^{2}$. The form is equal to zero only if $x=0$ and $\varphi\left(c^{\prime} x\right)=0$ if and only if $x=0$. Thus, $\dot{U}_{(16)}(t, x)$ is negative definite in the variable $x$.

From (i) and (ii) in (15) we see that $V(t, x)>0$ and $\dot{V}_{(13)}(t, x)<0$ for $x \neq 0$ and therefore $\dot{V}_{(16)}(t, x)$ is definitely nonzero on the set $x=0$. Hence, by Theorem 3.1 it follows that the set $H=\{0\}$ and the solution on $x=0$ of (16) is totally stable.

In the following theorem we make use of a converse theorem due to Malkin [26]. We say that the quadratic form $x^{\prime} C(t) x$ has the property $\mathrm{P}$ if it is positive definite and if the matrix $C(t)$ is uniformly bounded on $[0, \infty)$. Thus $x^{\prime} C(t) x$ has the property $\mathrm{P}$ if and only if there exist positive constants $c_{1}$ and $c_{2}$ such that

$$
c_{1}|x|^{2} \leq x^{\prime} C(t) x \leq c_{2}|x|^{2}, \quad t \geq 0
$$


Malkin has proved that if the system (13) is uniformly asymptotically stable and if $A(t)$ is uniformly bounded on $[0, \infty)$, then corresponding to each quadratic form $x^{\prime} C(t) x$ with property $\mathrm{P}$ there exists a quadratic form $V(t, x)=x^{\prime} D(t) x$ possessing property $\mathrm{P}$ such that $\dot{V}_{(13)}(t, x)=-x^{\prime} C(t) x$. And in fact the following formula determines $V(t, x)$.

$$
V(t, x)=x^{\prime} D(t) x=\int_{t}^{\infty}\left[X(s) X^{-1}(t) x\right]^{\prime} C(s)\left[X(s) X^{-1}(t) x\right] d s,
$$

where $X(t)$ is the fundamental matrix solution of (13).

THEOREM 4.2. Let $|A(t)| \leq A$ uniformly on $[0, \infty), \varphi \in F$ and $c^{\prime} b=-d<0$. Let $x^{\prime} C(t) x$ be a quadratic form such that (21) holds. Furthermore, suppose system (13) is uniformly asymptotically stable. Then, if $c_{1} d>\left(c_{2} L^{2}|b| / 2 \alpha+|c| A / 2\right)^{2}$, the zero solution of (16) is totally stable.

Proof. The theorem of Malkin guarantees the existence of $V(t, x)$ given by (22) and possessing property P. Using (14) and (21) we obtain from (22) that $|V(t, x)| \leq c_{2} L^{2}|x|^{2} / 2 \alpha$. A simple calculation shows that

$$
\begin{aligned}
\dot{V}_{(16)}(t, x) & =-x^{\prime} C(t) x+2 b^{\prime} D(t) x \varphi(\sigma) \\
& \leq-c_{1}|x|^{2}+c_{2} L^{2}|b||x||\varphi(\sigma)| / \alpha .
\end{aligned}
$$

Define the function $U(t, x)=V(t, x)+\Phi(x)$, where $\Phi(x)=\int_{0}^{\sigma} \varphi(s) d s, \sigma=c^{\prime} x$. This function is positive definite, radially unbounded and has an infinitesimal upper bound. Differentiating $U(t, x)$ along the solutions of (16) and using (18) and (23) yields

$$
\dot{U}_{(16)}(t, x) \leq-c_{1}|x|^{2}+2|x|\left(c_{2} L^{2}|b| / 2 \alpha+|c| A / 2\right)|\varphi(\sigma)|-d \varphi^{2}(\sigma) .
$$

The condition for the quadratic form in $|x|$ and $|\varphi(\sigma)|$ to be negative definite is $c_{1} d>\left(c_{2} L^{2}|b| / 2 \alpha+|c| A / 2\right)^{2}$. This form is equal to zero only if $x=0$ and $\varphi\left(c^{\prime} x\right)=0$ if and only if $x=0$. On the other hand, $V(t, x)>0$ and $\dot{V}_{(13)}(t, x)<0$ for $x \neq 0$ and therefore $\dot{V}_{(16)}(t, x)$ is definitely nonzero on the set $x=0$. Then by virtue of Theorem 3.1 it follows that the solution $x=0$ of (16) is totally stable.

Our last objective is to extend the above theorems to nonlinear plant equations. Consider the system

$$
\dot{x}=p(t, x),
$$

where $x$ and $p$ are vectors in $R^{n}, p(t, 0)=0$ and $p(t, x)$ is Lipschitzian in $x$ uniformly with respect to $t$. Suppose that the zero solution of (24) is globally exponentially stable in variation, i.e., there exist positive constants $\beta$ and $R$ such that

$$
\left|E\left(t, t_{0}, x_{0}\right)\right| \leq R e^{-\beta\left(t-t_{0}\right)}
$$

for all $t \geq t_{0} \geq 0$ and all $x_{0}$ in $R^{n}$, where $E\left(t, t_{0}, x_{0}\right)=\partial p\left(t, t_{0}, x_{0}\right) \partial x_{0}$ for a solution $p\left(t, t_{0}, x_{0}\right)$ of $(24)$ with $p\left(t_{0}, t_{0}, x_{0}\right)=x_{0}$. Then by Theorem 2.4 in [6] there exists a continuous scalar function $V$ on $[0, \infty) \times R^{n}$ such that

(i) $|x| \leq V(t, x) \leq R|x|$,

(ii) $\dot{V}_{(24)}(t, x) \leq-\beta V(t, x)$,

(iii) $|V(t, x)-V(t, y)| \leq R|x-y|$ 
for all $t \geq 0$ and $x, y$ in $R^{n}$. It seems that the global exponential stability in variation introduced in [6] is the most important concept of asymptotic stability. For details see Dannan and Elaydi [27].

Consider now the nonautonomous direct control system

$$
\dot{x}=p(t, x)+b \varphi(\sigma), \quad \sigma=c^{\prime} x,
$$

and its perturbed system

$$
\dot{x}=p(t, x)+b \varphi(\sigma)+g(t, x), \quad \sigma=c^{\prime} x,
$$

where $g(t, x)$ and the letters have the same meaning as in the previous case.

THEOREM 4.3. Let, for (24), $p(t, x)$ be Lipschitzian in $x$ uniformly relative to $t$ with a Lipschitz constant $Q$ and $x=0$ be globally exponentially stable in variation. Let $\varphi \in F$ and $c^{\prime} b=-d<0$. Then, if $4 \beta d>\left(R^{2}|b|+Q|c|\right)^{2}$, the zero solution of (26) is totally stable.

ProOF. The derivative of $\sigma$ along the solutions of (26) is

$$
\dot{\sigma}=c^{\prime} \dot{x}=c^{\prime} p(t, x)-d \varphi(\sigma) .
$$

Let $V(t, x)$ be the function given by Theorem 2.4 in [6]. This function satisfies the conditions (25). Its derivative along the solutions of (26) satisfies

$$
\dot{V}_{(26)}(t, x)=\dot{V}_{(24)}(t, x)+R|b \varphi(\sigma)|
$$

which because of (i) and (ii) in (25) becomes

$$
\dot{V}_{(26)}(t, x) \leq-\beta|x|+R|b||\varphi(\sigma)| \text {. }
$$

Consider the function $\Phi(x)=\int_{0}^{\sigma} \varphi(s) d s, \sigma=c^{\prime} x$. Then

$$
\dot{\Phi}_{(26)}(x)=\dot{\sigma} \varphi(\sigma)-c^{\prime} p(t, x) \varphi(\sigma)-d \varphi^{2}(\sigma) .
$$

Define the function $U(t, x)=V^{2}(t, x) / 2+\Phi(x)$. This function is positive definite, radially unbounded and has an infinitesimal upper bound. Using (25), (28), (29) and since $|p(t, x)| \leq Q|x|$ we obtain

$$
\dot{U}_{(26)}(t, x) \leq-\beta|x|^{2}+|x|\left(R^{2}|b|+Q|c|\right)|\varphi(\sigma)|-d \varphi^{2}(\sigma) .
$$

The condition for the quadratic form in $|x|$ and $|\varphi(\sigma)|$ on the right-hand side to be negative definite is $4 \beta d>\left(R^{2}|b|+Q|c|\right)^{2}$. Moreover, $\dot{U}_{(26)}(t, x)=0$ only if $x=0$ and $\varphi\left(c^{\prime} x\right)=0$ if and only if $x=0$. But $\dot{V}_{(26)}(t, x)$ is definitely nonzero on the set $x=0$ and then, by Theorem 3.1, the conclusion of the theorem follows.

We conclude this analysis by making a simple but important observation on the significance of the established results. In Theorems 4.1, 4.2 and 4.3, the function $U(t, x)$ is positive definite, radially unbounded and has an infinitesimal upper bound. Moreover, its derivatives along the solutions of (16) and (26) are negative definite. Then, by Theorem 22 of Massera [25], it follows that $x=0$ is uniformly asymptotic in the large for (16) and (26). But, since $\sigma=c^{\prime} x$, the same is true for $\sigma=0$ and therefore the systems (16) and (26) are absolutely stable. 
ACKNOWLEDGMENT. Aside from a generosity of my family, this research has not been financially supported by any public or private institution.

\section{REFERENCES}

1. A. M. Liapounoff [Liapunov], Problème général de la stabilité du mouvement, Ann. of Math. Studies, no. 17, Princeton Univ. Press, Princeton, N. J., 1949.

2. J. P. LaSalle, The stability of dynamical systems, CBMS Regional Conf. Ser. Appl. Math., no. 25, SIAM, Philadelphia, Pa., 1976.

3. V. M. Matrosov, On the stability of motion, Prikl. Mat. Mekh. 26 (1962), 885-895.

4. N. Rouche, P. Habets and M. Laloy, Stability theory by Liapunov's direct method, Appl. Math. Sci. 22, Springer-Verlag, Berlin, 1977.

5. G. N. Duboshin, The problem of the stability of motion under persistently acting perturbations, Trudy Gos. Astr. Inst. Sternberg 14 (1940), no. 1.

6. Z. S. Athanassov, Perturbation theorems for nonlinear systems of ordinary differential equations, J. Math. Anal. Appl. 86 (1982), 194-207.

7. _ On the asymptotic behaviour of nonlinear systems of ordinary differential equations, Glasgow Math. J. 26 (1985), 161-170.

8. __ Existence, uniqueness, and stability theorems for bounded solutions of nonlinear differential systems. Ann. Mat. Pura Appl. (4) 140 (1985), 301-330.

9. F. Brauer, Some stability and perturbation problems for differential and integral equations, Departamento de Matematica, Universidade de Brasilia, Brasilia, 1974.

10. W. Hahn, Theory and application of Liapunov's direct method, Prentice-Hall, Englewood Cliffs, N.J., 1963.

11. V. I. Zubov, The methods of Liapunov and their applications, Noordhoff, Groningen, 1964.

12. N. P. Bhatia and G. P. Szegö, Stability theory of dynamical systems, Springer-Verlag, Berlin, 1970.

13. A. A. Sestakov, Tests for the instability of a set with respect to a nonautonomous differential system, Differencial'nye Uravnenija 13 (1977), 958-960.

14. _ Criteria for the stability of sets with respect to a nonautonomous differential system, Differential'nye Uravnenija 13 (1977), 1079-1090.

15. J. P. LaSalle and G. Szegö, Comments on L. Hwang's paper "New methods for constructing Liapunov functions for time-invariant control systems", Proc. Second Congr. IFAC, Butterworths, London, 1964, p. 582.

16. J. P. LaSalle, Recent advances in Liapunov stability theory, SIAM Rev. 6 (1964), 1-11.

17. T. Yoshizawa, Eventual properties and quasi-asymptotic stability of a non-compact set, Funkcial. Ekvac. 8 (1966), 79-90.

18. O. Hájek, Absolute stability of noncompact sets, J. Differential Equations 9 (1971), 496508.

19. , Ordinary and asymptotic stability of noncompact sets, J. Differential Equations 11 (1972), 49-65.

20. I. G. Malkin, Stability in the case of constantly acting disturbances, Prikl. Mat. Mekh. 8 (1944), 241-245.

21. S. I. Gorshin, On the stability of motion under constantly acting perturbations, Izv. Akad. Nauk Kazakh. SSR Ser. Mat. Mekh. 256 (1948), 46-73.

22. S. Lefschetz, Stability of nonlinear control systems, Academic Press, New York, 1965.

23. M. A. Aizerman and F. R. Gantmakher, Absolute stability of regulator systems, HoldenDay, San Francisco, Calif., 1964. 
24. I. G. Malkin, On the reversibility of Liapunov's theorem on asymptotic stability, Prikl. Mat. Mekh. 18 (1954), 129-138.

25. J. L. Massera, Contributions to stability theory, Ann. of Math. (2) 64 (1956), 182-206.

26. I. G. Malkin, On the construction of Liapunov functions for systems of linear equations, Prikl. Mat. Mekh. 16 (1952), 239-242.

27. F. M. Dannan and S. Elaydi, Lipschitz stability of nonlinear systems of differential equations, J. Math. Anal. Appl. (to appear).

INSTITUte of Mathematics, Polish ACADEMY of Sciences, 00-950 WARSAW, SNIADECKICH 8, POLAND 